\title{
Modelo de sistematización en los proyectos sociales de ApS (UIC)
}

\author{
María Teresa Fuertes CAmacho \\ Universitat Internacional de Catalunya \\ tfuertes@uic.es
}

\begin{abstract}
Resumen:
La metodología del aprendizaje-servicio (ApS) responde a las necesidades derivadas del contexto social y universitario actual y favorece la doble misión académica y social que debe cumplir la institución universitaria. Las distintas fases seguidas para llevar a cabo los distintos proyectos sociales implementados en el Practicum de Grado de Educación de la Universitat Internacional de Catalunya (UIC) resultaron claves para la puesta en práctica de competencias transversales imprescindibles para el trabajo y para la vida de los estudiantes que participaron en la experiencia.
\end{abstract}

Palabras Clave: Aprendizaje-Servicio; Practicum; responsabilidad social universitaria, educación; competencias transversales

\section{Model of systematization in Service- Learning Projects (UIC)}

\begin{abstract}
:
The Service-Learning methodology responds to the needs of social context and promotes academic and social dual mission to be met by the universities. The different phases followed to carry out the projects implemented during the Internships of Education Degree at the Universitat Internacional de Catalunya (UIC) were key to the implementation of generic skills essential for work and for life-long learning.
\end{abstract}

Key Words: Service-Learning; Internships; social responsibility; skills: teaching education

\section{Referencia normalizada:}

Fuertes Camacho, M. T. (2014): Modelo de sistematización en los proyectos sociales de ApS (UIC). Historia y Comunicación Social. Vol. 19. Núm. Especial Marzo. Págs. 175-186.

Sumario: 1. Introducción. 1.1. Formación por competencias en la educación superior. 2. Metodología. 2.1. Fases de implementación. 2.2. Campos y contextos de actuación. 2.3. Instrumento de evaluación. 2.4. Análisis de la información. 3.Conclusiones. 4.Referencias bibliográficas.

\section{Introducción}

La sociedad pide cambios en la educación y estos cambios en los diferentes niveles educativos requieren de maestros y de maestras preparados, con una sólida formación inicial, capaces de tomar decisiones innovadoras, de trabajar en equipo y de relacionarse con otros colectivos como familias, asociaciones y entidades, con una 
preocupación por la formación continua que les permita la adaptación profesional a una sociedad de cambios constantes. No se trata solamente de cambiar contenidos, sino de cambiar las formas de enseñar que permitan acceder a ellos. Un estudiante que ya posee determinado conocimiento y que ya ha realizado determinada actividad, hasta que no se enfrente a una situación académica o profesional determinada no revelará su nivel de competencia (De Miguel, 2005). La formación académica y la formación práctica deben ser complementarias y retroalimentarse de manera continua puesto que una no existe sin la otra (Álvarez Rateike, 2011). El Prácticum supone la inmersión de los estudiantes, durante determinado periodos en centros educativos con la finalidad de conocerlos directamente, integrar los conocimientos teóricos y prácticos, las maneras de hacer, las actitudes y los valores que se viven en contextos reales. El Prácticum debe permitir, por una parte la relación entre el conocimiento teórico y el conocimiento práctico y, al mismo tiempo, debe hacer posible la creación de nuevo conocimiento a partir de la práctica (Cochran-Smith, 2002).

Aunque la mayor parte del proceso se desarrolle lejos de la universidad, el tutor de la universidad deberá conocer el entorno de aprendizaje y mantener el contacto con el centro y en concreto con el tutor del centro colaborador de prácticas. Disponer de profesorado con experiencia en Prácticum es una condición imprescindible para que las actuaciones sean adecuadas a las distintas situaciones y representen una colaboración y una coordinación real con los centros.

Antes de concretar las competencias, se deben tener en cuenta toda una serie de consideraciones respecto al Prácticum, entre las cuales hemos considerado oportuno, por el objetivo de nuestro trabajo, destacar las siguientes:

El Prácticum es un componente del currículum formativo que se encuentra comprometido (como el resto de los componentes curriculares) con el refuerzo de las competencias profesionales (conocimientos + habilidades + actitudes) ya iniciadas en el trabajo institucional y con la adquisición de otras nuevas (propias del periodo de prácticas). Las competencias no solamente pertenecen al rango de los aprendizajes operativos y prácticos... sino que cubren un espectro de dimensiones más amplio (las llamadas competencias del tercer nivel, que implican un dominio superior de la actividad profesional tales como los aspectos técnicos o de gestión, la motivación personal y la relación con los demás, la creatividad, la capacidad de analizar críticamente el propio trabajo y el compromiso social y ético, entre otros) (Zabalza, 2006:7-8).

Estas competencias a las que Zabalza denomina del "tercer nivel "nos interesan especialmente puesto que son competencias transversales que resultan imprescindibles para llevar a cabo con éxito los proyectos de aprendizaje servicio.

\subsection{Formación por competencias en la educación superior}

La competencia ha pasado a aplicarse al proceso formativo de los estudiantes en el marco de la educación superior y las posibilidades y las alternativas teóricas y paradigmáticas para diseñar y tipificar estas competencias pueden ser diversas. Independientemente del documento inicial que inspire su concreción, la competencia debe 
tener un carácter integrador que abarque los tres ámbitos de aprendizaje: conocer, actuar (procedimientos) y ser en relación a las disposiciones y actitudes personales, sociales, académicas, científicas o profesionales (Martínez Ruíz \& Sauleda, 2007:31).

En el contexto europeo, existen tres documentos considerados relevantes para establecer un marco de definición para el diseño de los planes de estudio basados en el desarrollo competencial de los estudiantes: el informe Delors, el proyecto Tuning y el proyecto DeSeCo elaborado por la OCDE.

A petición de la UNESCO, el informe para la educación del siglo XXI fue elaborado por una comisión internacional de personas pertenecientes al mundo docente y fue presidia por Delors. La educación a lo largo de la vida, según el citado el informe, se basa en cuatro pilares: aprender a conocer, aprender a hacer, aprender a vivir juntos y aprender a ser.

Eso que proponemos supone trascender la visión puramente instrumental de la educación considerada como la vía necesaria para obtener resultados (dinero, carreras, etc.) y supone cambiar para considerar la función que tiene en su globalidad la educación. La realización de la persona, que toda entera debe aprender a ser (Delors, 1997:74)

El informe Delors hace hincapié en la importancia de la apertura de la mente hacia otras culturas, otras civilizaciones y otras experiencias. Cada miembro de la colectividad debe asumir su responsabilidad para con los demás de forma cotidiana, en su actividad profesional, cultural, asociativa y de consumidor y para ello, hay que preparar a cada persona para esa participación, enseñándole sus derechos y sus deberes, y desarrollando sus competencias sociales.

Uno de los más claros ejemplos de conceptualización de las competencias en el marco europeo lo representa el proyecto Tuning iniciado en el año 2001. El proyecto contó con la participación de ciento treinta y cinco universidades europeas y se desarrolló gracias a la financiación de la Comisión Europea, con el objetivo de seguir el proceso de implementación de la formación de la Declaración de Bolonia en la universidad.

La formación debe ir mucho más allá del empleo, puesto que debe suponer también la preparación de los estudiantes para integrarse en la sociedad. El trabajo recogido en el Proyecto Tuning pretendía ser un incentivo para las universidades para desarrollar sus estrategias no solamente en relación a los saberes vinculados a los conocimientos o a los contenidos, sino también en relación a las competencias genéricas y específicas del proceso de enseñanza y aprendizaje. Como consecuencia, se estableció la clasificación de las competencias en específicas y transversales.

No se trata de mejorar solamente la preparación profesional de los estudiantes para ocupar un determinado puesto laboral, sino principalmente ofrecer una formación más sólida, más firme, más acorde con el enfoque de lo que debe ser una buena formación universitaria, que ayude a los estudiantes a saber, saber hacer, saber convivir y saber ser. 
Desde la perspectiva de educadores, las competencias deben estar enraizadas en las motivaciones, en las actitudes y en los valores que marcarán la direccionalidad de las actividades profesionales de las personas formadas en la Universidad (Villa \& Poblete, 2004:11).

La incorporación de las competencias genéricas al currículum académico es un reto más de los que se nos presentan en el proyecto de convergencia europeo y forzosamente en el proceso de innovación pedagógica que el proyecto supone. Incorporar las competencias a nuestra actividad docente implica pasar de la formación de especialistas cualificados en una materia concreta a la educación de personas profesionalmente competentes, que den respuesta a necesidades de la sociedad.

El proyecto Definition and Selection of Competences (DeSeCo), elaborado por la OCDE con la colaboración de la UNESCO y de expertos e instituciones, para identificó un conjunto reducido de competencias clave. El marco conceptual del Proyecto DeSeCo clasifica las competencias clave en tres amplias categorías interrelacionadas entre sí: una primera que capacita para el uso de una amplia gama de herramientas que permitan actúan de manera interactiva, una segunda categoría que permita poder comunicarse con otros y actuar en grupos heterogéneos; y finalmente, una tercera categoría que capacite para ejercer la responsabilidad situando sus vidas en un contexto social más amplio.

Si establecemos un paralelismo entre esta clasificación y la establecida en Tuning, la primera categoría del DeSeCo coincidiría con las competencias genéricas instrumentales del Tuning, la segunda con las genéricas de carácter interpersonal, y la tercera categoría con las competencias genéricas de carácter sistémico. Las herramientas adquiridas mediante las competencias de la categoría 1, ayudan al desarrollo de las competencias de las categorías 2 y 3 y todas, en conjunto afectan a la dimensión personal y social.

De acuerdo con el documento:

Una competencia también es un factor importante en la contribución de los individuos a cambiar o transformar el mundo, no sólo la forma en que le hacen frente. Así como las competencias se relacionan con las características principales y las demandas de la vida moderna, también están determinadas por la naturaleza de nuestras metas, tanto como individuos y como sociedad (OCDE, 2002:5).

Las competencias deberían responder a objetivos individuales y colectivos y representar un éxito no solamente para el propio individuo, sino también para la sociedad y favorecer de esta manera el bien común. Según Perrenoud (2001), quien llega a dominar una determinada competencia es porque dispone a la vez de los conocimientos necesarios y de la capacidad de movilizarlos para definir y solucionar verdaderos problemas.

Le Boterf (2001:121) caracteriza al profesional "como aquel que sabe gestionar y manejar una situación profesional compleja" lo que supone: saber actuar y reaccionar con pertinencia, saber combinar los recursos y movilizarlos en un contexto, saber transferir, saber aprender y aprender a aprender; y saber comprometerse. La combi- 
nación de los mencionados saberes definiría la competencia que no puede entenderse como un conocimiento estático imposible de reducir a un saber o a un saber hacer.

Barnett (2008) señala cuatro condiciones para poder hablar del desarrollo competencial en la formación universitaria. Las condiciones que propone son las siguientes: existencia de una situación con cierta complejidad, desempeño que considere la situación y que sea intencional, evaluación de la actuación que constate la superación de las demandas establecidas y sensación de trabajo bien hecho. Las competencias genéricas según Rychen (2002) a parte de multifuncionales, son transversales puesto que pueden aplicarse a cualquier campo social, se refieren a un orden superior de complejidad mental puesto que favorecen el desarrollo de habilidades intelectuales e impulsan el crecimiento de actitudes y valores profundos (Villa \& Poblete, 2007:45). Al mismo tiempo son también multidimensionales puesto que afectan el desarrollo de diferentes dimensiones (personal, social, académico, práctico e institucional).

El objetivo de nuestra investigación se centra en aportar datos relativos al impacto que los programas de aprendizaje servicio tienen en el desarrollo de determinadas competencias transversales claves para la formación inicial del profesorado, a partir de una propuesta de implementación que vamos a presentar en el siguiente apartado.

\section{Metodología}

El aprendizaje servicio es una estrategia de enseñanza y de aprendizaje experiencial que permite aprender, desarrollar y demostrar competencias en la acción, mediante la planificación y la implementación de proyectos que dan respuesta a necesidades reales del contexto donde se actúa. Su puesta en práctica implica la participación activa de los estudiantes y la coordinación entre instituciones que colaboran con el objetivo de integrar objetivos de aprendizaje y objetivos de servicio que favorezcan el desarrollo académico- social y de superación personal de los estudiantes que aprenden a emprender acciones en beneficio de la comunidad. Si bien el aprendizaje experiencial, activo y relacionado con la comunidad no es nuevo, se debe reconocer que, desde el enfoque del ApS, la colaboración y la participación implican un proceso de sistematización y de seguimiento de unas fases que no existía previamente.

\subsection{Fases de implementación}

La implementación de los proyectos de aprendizaje-servicio sigue tres grandes fases: fase previa o de planificación; fase de ejecución, durante la estancia de prácticas; y fase posterior o de evaluación. La reflexión acompaña todo este proceso de investigación - acción. Cada una de estas fases incluye un conjunto de acciones que se recogen que se llevan a cabo durante el curso académico: 
Figura 1: Fases y subfases de los proyectos ApS implementados.

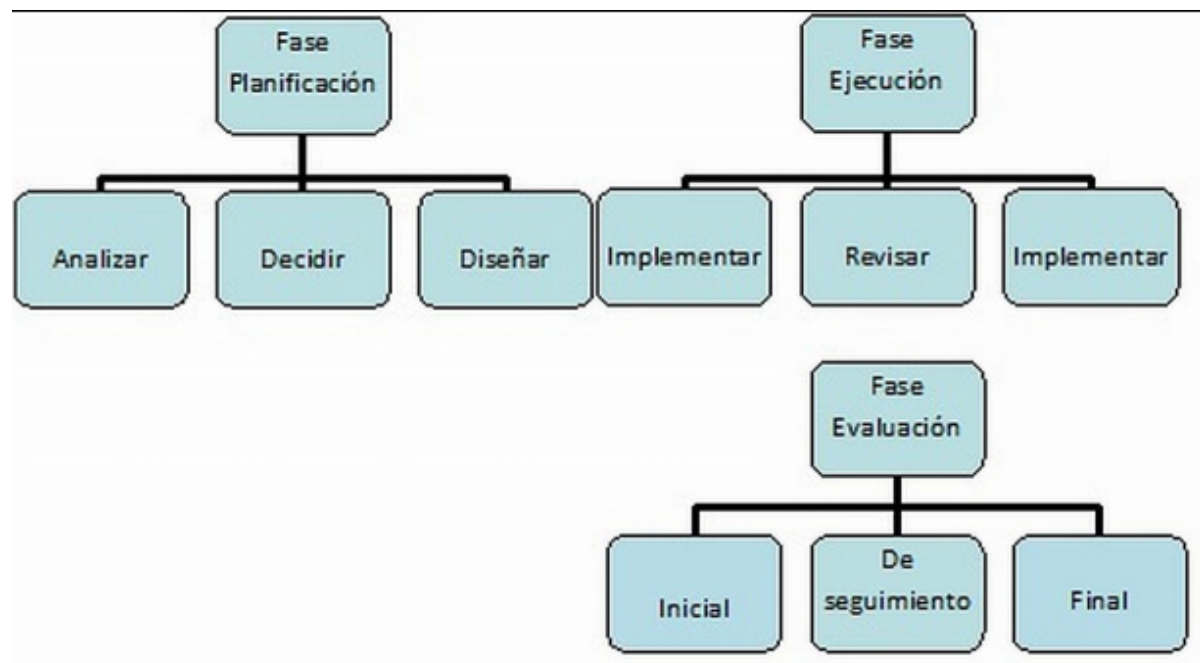

Fuente: Adaptación García (2006)

Cada una de las tres fases incluye diferentes acciones. En todas ellas resulta imprescindible la reflexión que el estudiante llevara a cabo con la ayuda de los tutores de la universidad y del centro. La fase de ejecución implica un proceso constante de investigación - acción. La fase de evaluación la situamos gráficamente entre las fases de panificación y de ejecución puesto que ya se lleva a cabo una evaluación inicial al detectar las necesidades y la concreción de la actuación que se va a llevar a cabo en base a las necesidades y las posibilidades de los estudiantes. La evaluación está presente también durante la ejecución del proyecto y al final del mismo.

\subsection{Campos y contextos de actuación}

Para la puesta en práctica de los proyectos se han tenido en consideración los campos de actuación definidos por Bosch y Batlle (2006):

Figura 2: Relación entre los ámbitos de actuación y los proyectos ApS desarrollados en los contextos de prácticas.

\begin{tabular}{|l|l|l|}
\hline \multicolumn{1}{|c|}{ Campos de actuación } & Proyectos ApS ejecutados & Contextos de prácticas \\
\hline $\begin{array}{l}\text { Mejora del medio ambiente: acciones } \\
\text { de sensibilización en defensa de la } \\
\text { naturaleza y la sostenibilidad. }\end{array}$ & Medio ambiente & $\begin{array}{l}\text { Escuelas rurales de } \\
\text { Guatemala }\end{array}$ \\
\hline
\end{tabular}




\begin{tabular}{|c|c|c|}
\hline $\begin{array}{l}\text { Atención a personas y a colectivos } \\
\text { cercanos con dificultades: } \\
\text { intervenciones con niños y niñas de } \\
\text { entornos desfavorecidos; personas } \\
\text { enfermas, o en riesgo de exclusión } \\
\text { o personas necesitadas de apoyo } \\
\text { formativo. }\end{array}$ & $\begin{array}{l}\text { Dinámicas de trabajo en } \\
\text { equipo } \\
\text { Material didáctico } \\
\text { de lectoescritura y } \\
\text { matemáticas } \\
\text { Integración de las } \\
\text { familias en el programa } \\
\text { formativo } \\
\text { Taller de expresión y } \\
\text { comunicación }\end{array}$ & $\begin{array}{l}\text { Escuelas rurales de } \\
\text { Guatemala } \\
\text { Aulas de los centros } \\
\text { hospitalarios } \\
\begin{array}{l}\text { Escuelas en contextos } \\
\text { desfavorecidos }\end{array}\end{array}$ \\
\hline $\begin{array}{l}\text { Mejora de la calidad de vida } \\
\text { y participación ciudadana: } \\
\text { dinamización cultural }\end{array}$ & $\begin{array}{l}\text { Biblioteca escolar } \\
\text { Animación a la lectura } \\
\text { Intercambio cultural }\end{array}$ & $\begin{array}{l}\text { Escuelas rurales de } \\
\text { Guatemala } \\
\text { Aulas de los centros } \\
\text { hospitalarios }\end{array}$ \\
\hline $\begin{array}{l}\text { Defensa de los derechos humanos } \\
\text { (derecho a la educación). }\end{array}$ & $\begin{array}{l}\text { Evitar el abandono } \\
\text { escolar de las niñas }\end{array}$ & $\begin{array}{l}\text { Escuelas rurales de } \\
\text { Guatemala }\end{array}$ \\
\hline
\end{tabular}

Fuente: Elaboración propia

Se llevaron a cabo diez proyectos en tres contextos de prácticas distintos y participaron en la experiencia veinticuatro estudiantes del último curso de Educación. Los objetivos que define el aprendizaje - servicio son básicamente tres: mejorar la calidad del aprendizaje establecido manteniendo niveles de exigencia académica, los objetivos de servicio, que se traducen en un servicio o producto de calidad que aporte solución a alguna problemática social real y la formación en valores de los estudiantes.

\subsection{Instrumento de evaluación}

Al finalizar los proyectos, aplicamos un cuestionario para evaluar el impacto en el desarrollo de siete competencias transversales consideradas clave que analizaremos en el siguiente apartado.

En las entrevistas los estudiantes encuestados expresan sus propias perspectivas personales (Patton, 1984). Si el objeto evaluado forma parte del mismo agente evaluador, como ocurre en nuestro caso, hablamos de "autoevaluación", Martínez Olmo (2004). Los estudiantes valoraron el grado de adquisición de las competencias después de seguir las fases y el esquema de los proyectos de ApS que hemos visto anteriormente. El baremo utilizado fue: 0 (No sé), 1 (Nada), 2(Poco), 3 (Bastante), 4 (Mucho).

Este tipo de evaluación responde, según Boud (1995), a un concepto de educación más ligado a los objetivos de aprender a aprender, a reflexionar y a emanciparse, donde el centro de la educación se sitúa más en el propio individuo o institución que aprende. 


\subsection{Análisis de la información}

Las siete competencias transversales se seleccionaron en base a las investigaciones realizadas en EEUU sobe las competencias transversales que se adquieren al realizar proyectos ApS y se recogen en la tabla siguiente:

Figura 3:Competencias evaluadas e indicadores evaluados.

\begin{tabular}{|l|c|l|}
\hline \multicolumn{1}{|c|}{ Competencia evaluada } & Código & \multicolumn{1}{|c|}{ Indicador (resultado de aprendizaje) } \\
\hline $\begin{array}{l}\text { Adaptación al entorno y } \\
\text { tratamiento de las adversidades. }\end{array}$ & $\mathrm{C} 1$ & $\begin{array}{l}\text { Mantenerse dinámico/a y activo/a para } \\
\text { continuar realizando tareas a pesar las } \\
\text { dificultades. }\end{array}$ \\
\hline Planificación y acción: & $\mathrm{C} 2$ & $\begin{array}{l}\text { Participar e integrarse en el desarrollo } \\
\text { organizado de un trabajo con los demás, } \\
\text { haciendo la previsión de las tareas, tiempos } \\
\text { y recursos necesarios para conseguir los } \\
\text { resultados deseados. }\end{array}$ \\
\hline Toma de decisiones & $\mathrm{C} 3$ & $\begin{array}{l}\text { Colaborar con los demás para tomar } \\
\text { decisiones conjuntas de calidad. }\end{array}$ \\
\hline Responsabilidad social & $\mathrm{C} 4$ & $\begin{array}{l}\text { Actuar con responsabilidad y con el } \\
\text { compromiso que supone la acción orientada } \\
\text { ala comunidad. }\end{array}$ \\
\hline Pensamiento crítico & C6 & $\begin{array}{l}\text { Hacerse preguntas sobre la realidad que } \\
\text { nos envuelve, considerando los juicios y } \\
\text { reflexionando sobre las consecuencias de las } \\
\text { acciones propias y ajenas }\end{array}$ \\
\hline Comunicación interpersonal & $\begin{array}{l}\text { Hacer uso del diálogo y el entendimiento } \\
\text { para colaborar y generar buenas relaciones } \\
\text { con otras personas. }\end{array}$ \\
\hline Resolución de problemas & C7 & $\begin{array}{l}\text { Utilizar la experiencia y el criterio para analizar } \\
\text { las causas de un problema y construir una } \\
\text { solución. }\end{array}$ \\
\hline
\end{tabular}

Fuente: Elaboración propia

Los resultados obtenidos se reflejan en el siguiente gráfico que muestra un alto porcentaje de respuestas positivas en todas las competencias transversales evaluadas tanto en las de carácter instrumental: planificación y acción, resolución de problemas y toma de decisiones; interpersonal: comunicación interpersonal, pensamiento crítico y responsabilidad social; y sistémicas: adaptación al entorno y tratamiento a las adversidades. 
Figura 4. Resultados valoración de las competencias seleccionadas

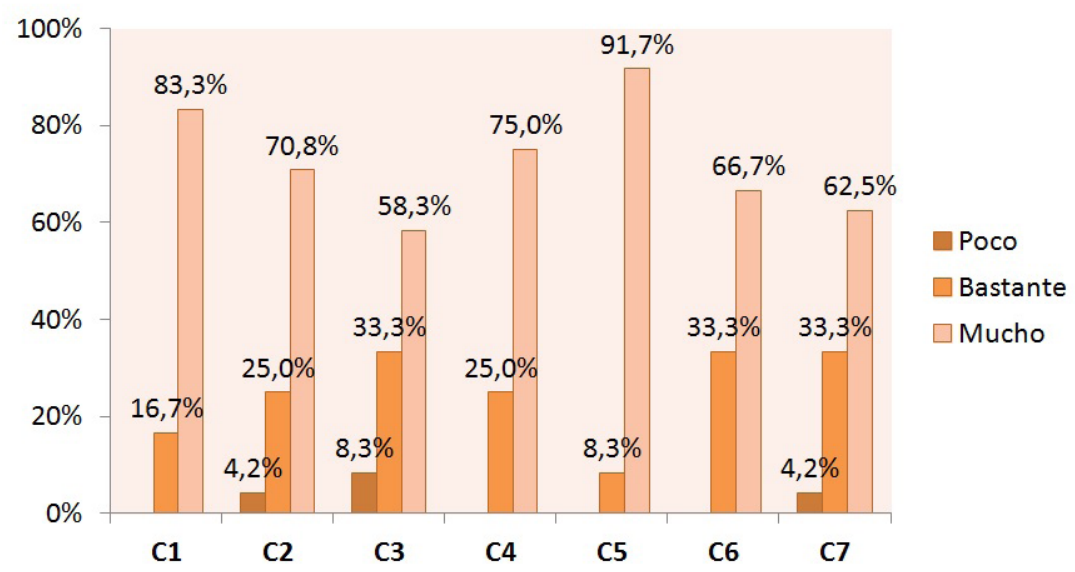

\section{Conclusiones}

La universidad no puede olvidar que tiene una misión intelectual y una misión social. La creación de un espacio de educación superior europea supone un cambio de paradigma de una educación centrada en la enseñanza, a una educación basada en el aprendizaje. Por ello, es preciso establecer cambios profundos sobre la organización del aprendizaje, el enfoque de las actividades y el papel del educador (González y Wagenaar, 2003).

En el aprendizaje servicio la participación de los estudiantes resulta fundamental para la puesta en práctica de competencias transversales imprescindibles más allá de la profesión. La metodología utilizada permite al estudiante involucrarse directamente en el contexto, adaptándose a sus necesidades y a una realidad que a menudo es muy diferente de la vivida en el aula (Folgueiras, Luna, \& Puig, 2011). El ApS involucra y relaciona al enseñante y al aprendiz combinando la experiencia personal y la sabiduría adquirida en la comunidad. Se basa en nuevas técnicas pedagógicas que van más allá de las tradicionales que tienen como base común que el estudiante construya su propio aprendizaje. El aprendizaje se pone a disposición del servicio y éste favorece, a su vez, el desarrollo integral del estudiante a través de la acción social. 


\section{Bibliografía}

\subsection{Libros:}

ANDER-EGG, E., Y AGUILAR, M. (1997). Cómo elaborar un proyecto. Guía para elaborar proyectos sociales y culturales. Buenos Aires: Editorial Lumne Humanitas.

BARNETT, R. (2008). Para una transformación de la Universidad. Nuevas relaciones entre investigación, saber y docencia. Barcelona: Octaedro.

BOUD, D. (1995). Enhancing learning through Self Assessment. Londres: Kogan Page.

DE MIGUEL, M. (2005). Modalidades de enseñanza centradas en el desarrollo de competencias. Orientaciones para promover el cambio metodológico en el Espacio Europeo de Educación Superior. Oviedo: Universidad de Oviedo.

DELORS, J. (1997). La educación encierra un tesoro Informe. Comisión Internacional sobre la educación para el siglo XXI. París: UNESCO.

GARCÍA, F. (2006). La intervención profesional en Trabajo Social. Supuestos prácticos I. Málaga: Colegio Oficial de Diplomados en Tarbajo Social.

GONZÁlEZ, J., Y WAGENAAR, R. (2003). Tuning Educational Structures in Europe. Informe Final. Fase, 1. Bilbao: Universidad de Deusto.

LE BOTERF, G. (2001). Ingeniería De Las Competencias Ediciones Gestión 2000. Barcelona: Ediciones Gestión 2000.

MARTÍNEZ RUÍZ, Ma A.;SAULEDA, N. (2007). Glosario EEES. Terminología relativa al Espacio Europeo de Educación Superior. Alcoy: Marfil S.A.

MATEO, J. (2009). Guía para la evaluación de competencias en el trabajo de fin de grado en el ámbito de la ciencias sociales y jurídicas. Barcelona: AQU.

OCDE. (2002). Definition and Selection of Competences (DESECO): Theoretical and Conceptual Foundations. París: OCDE.

PATTON, M. (1984). Qualitative Evaluation Methods. Beverly Hills: Sage. TAPIA, M.N. (2004). Aprendizaje y Servicio Solidario. Madrid. CcS. VILLA, A.; POBLETE, M. (2007). Aprendizaje basado en competencias. Una propuesta para la evaluación de las competencias genéricas. Bilbao: Ediciones Mensajero. UD.

4.2 Capítulos de libros:

FURCO, A. (2001). "Is Service-Learnin really better than Community Service? A study of high school service program outcomes". En A. FURCO, Y S. BILLING (2001). Service-Learnig. The essence of the pedagogy (pp. 23-49). Greenwich, Connecticut: Information Age Publishing Inc.

MARTÍNEZ OLMO, F. (2004). "La investigación evaluativa". En BISQUERRA, R.. Metodología de la investigación educativa. Madrid: La Muralla.p. 425-446. 


\subsection{Revistas en papel}

BOSCH, C., Y BATLLE, R. (2006). Proyectos para mejorar la ciudadanía. Cuadernos de Pedagogía, 357, p. 64-68.

COCHRAN-SMITH, M. (2002). Learning and unlearning: The education of teacher educators. Teachers and Teacher Education, 19, p. 5-28.

PERRENOUD, P. (2001). La formación de los docentes en el siglo XXI. Revista de Tecnología educativa XIV, 3, p. 503-523.

ZABALZA, M. (2006). Organitzar el Practicum en un model de competències. Perspectiva Escolar, 307, p. 2-16.

ZABALZA, M. (2006). Organitzar el Practicum en un model de competències. Perspectiva Escolar,307. p 2-16.

4.4 Artículos en publicaciones web:

AGUIRRE, C., ANDRADE, M., Y ZULUETA, S. Aprendizaje Servicio. Manual para docentes UC. Disponible en: http://creas.uahurtado. $\mathrm{cl} / \mathrm{html} /$ documentos/aprendizajeservicio/manualuc.pdf[16-10-2012]. ÁLVAREZ RATEIKE., J. (2011). Formación académica vs. formación práctica: la eterna disputa. Educaweb Monográfico 240. La excelencia universitaria: el compromiso con la calidad.

Dispopnible en: http://www.educaweb.com/noticia/2011/10/31/formacion-academica-vs-formacion-practica-eterna-disputa-5046/[24-05-2013].

VIDAL, S. FUERTES, M.T (2013) « La dinámica de grupos para el trabajo cooperativo facilita la comunicación» Vivat Academia, UCM, 123.

Disponible en: http://www.vivatacademia.net/index.php/vivat/article/view/1/2 . [18-07-2013].

4.5 Diarios:

La Vanguardia, 25-10-2013. El valor del esfuerzo

\section{La autora}

M. Teresa Fuertes Camacho. Profesora adjunta de la Facultad de Educación de la Universitat Internacional de Catalunya donde ocupa el cargo de Vicedecana de la Facultad y Subdirectora del Departamento de Educación. Doctora en Humanidades, Licenciada en Psicopedagogía, especialista en asesoramiento curricular y Diplomada en Magisterio. Miembro de la Red Universitaria Española de Aprendizaje -Servicio y Directora del Prácticum de Grado de Educación de la UIC. Miembro de la Comisión Interuniversitaria de Cataluña - Programa de Mejora e Innovación de la Formación Inicial del Profesorado. Sus líneas de investigación giran en torno a la profesionali- 
zación a través del Practicum y la responsabilidad social universitaria. Colaboradora en las investigaciones del grupo ROIP (Réseau Observatoire International sur la Professionnalisation) y SIRSU (Sostenibilidad y Responsabilidad Social Universitaria). 